\title{
Estimation of the Solution of the Kolmogorov- Fisher Type Biological Population Task by Taking Into Account the Reaction-Diffusion
}

\author{
Muhamediyeva Dildora Kabulovna
}

\begin{abstract}
The spatial-temporal dynamics of the population is one of the most interesting aspects and problems for environmental modeling. In this article, we will consider some mathematical models based on one-dimensional reaction-diffusion-advection equations for population growth in a heterogeneous habitat. Considering a number of models of increasing complexity, we investigate often the opposite roles of advection and diffusion for the conservation of the population.

Whenever possible, we demonstrate basic mathematical methods and provide critical conditions that ensure the survival of the population, in simple systems and in more complex resourceconsumer models.
\end{abstract}

Keywords: biological population, reaction-diffusion, crossdiffusion, parabolic system, quasilinear equations.

\section{INTRODUCTION}

In the 1930s Fisher and Kolmogorov, Petrovsky, Piskunov in population dynamics and Zel'dovich, Frank-Kamenetsky in combustion theory began to study problems with reaction conditions [1-2].

Population modeling is of great importance in ecology and economics, for example, for describing predator-prey interactions and competition, predicting the dynamics of cell divisions and infectious diseases, and for managing renewable resources (harvesting). Population models describe the change in the number of species due to fertility, mortality and movement from position to position (in space) or from stage to stage (age, size, etc.) [3-6]. In this brief review, we will look at some mathematical results for deterministic and continuous population models. We concentrate on the following classes of models:

spatially homogeneous population models;

- spatially heterogeneous population models;

- age and size demographic models; and

- models of the population with a delay in time.

The evolution of spatially homogeneous populations can be modeled by ordinary differential equations, for example, a logistic growth model.

Revised Manuscript Received on July 25, 2019

Muhamediyeva Dildora Kabulovna, Ph.D, Tashkent university of information technologies.
The interaction of competing populations can be described by a system of coupled equations, one of which is the well-known Volterra-Tray system [7]. Important issues, in addition to the justification of the relevant problems, are the stability of stable states and biological consequences. In spatially heterogeneous conditions, the population size varies in space and can spread in the environment. This gives a class of reaction-diffusion equations and their systems [8-9]. Turing found that a stationary solution of the diffusion system can become unstable even if the stable state of the corresponding system without diffusion is stable. Thus, stability analysis is much more involved than in the spatially homogeneous case. Roughly speaking, in the long run, extinction or coexistence of species may occur.

Solutions of the diffusion model of the Voltaire-Lotka competition do not show the formation of patterns. Consequently, this model is not capable of describing segregation phenomena. Analysis of the existence of cross-diffusion systems is difficult due to strong nonlinear coupling and since diffusion matrices can not be either symmetric or positive definite [7-8]. Recently, analytical tools have been developed that prove the existence of time-varying weak solutions. When the individuals of the population are not identical, but may differ in age, size, etc., we need to introduce structured population models.

The field of population modeling has become so large that in this review we can consider only a small part of the published model-mathematical topics. Many classes of models and important questions will not be discussed. For example, we ignore difference and matrix equations, stochastic approaches and models, including mutations, maturation structures, metapopulations, and demographic or biomedical applications.

\section{FORMULATION OF THE PROBLEM}

In this paper we investigate the properties of solutions of the problem of a biological population of the FisherKolmogorov type in the case of variable density. The main method of investigation is the selfsimilar approach. Consider 
Estimation of the Solution of the Kolmogorov-Fisher Type Biological Population Task by Taking Into Account the Reaction-Diffusion

in the field $\mathrm{Q}=\left\{(\mathrm{t}, \mathrm{x}): 0<\mathrm{t}<\infty, \mathrm{x} \in \mathrm{R}^{\mathrm{N}}\right\}$ parabolic system of two quasilinear reaction-diffusion equations

$$
\left\{\begin{array}{l}
\frac{\partial u_{1}}{\partial t}=\nabla\left(D_{1} u_{2}^{m_{1}-1}\left|\nabla u_{1}^{k}\right|^{p-2} \nabla u_{1}\right)+k_{1} u_{1}\left(1-u_{1}^{\beta_{1}}\right), \\
\frac{\partial u_{2}}{\partial t}=\nabla\left(D_{2} u_{1}^{m_{2}-1}\left|\nabla u_{1}^{k}\right|^{p-2} \nabla u_{2}\right)+k_{2} u_{2}\left(1-u_{2}^{\beta_{2}}\right),
\end{array}\right.
$$

$\left.u_{1}\right|_{t=0}=u_{10}(x),\left.u_{2}\right|_{t=0}=u_{20}(x)$,

which describes the process of a biological population of the Kolmogorov-Fisher type in a nonlinear two-component medium, the coefficients of mutual diffusion are respectively equal

$$
D_{1} u_{2}^{m_{1}-1}\left|\nabla u_{1}^{k}\right|^{p-2} \nabla u_{1},
$$

$D_{2} u_{1}^{m_{2}-1}\left|\nabla u_{2}^{k}\right|^{p-2} \nabla u_{2} \quad$. Numeric $\quad$ parameters $m_{1}, m_{2}, n, p, \beta_{1}, \beta_{2}, D_{1}, D_{2}$ - positive real numbers, $\nabla()-.\underset{x}{\operatorname{grad}(.)}, \beta_{1}, \beta_{2} \geq 1$, $x \in R^{N} l>0$;

$u_{1}=u_{1}(t, x) \geq 0, u_{2}=u_{2}(t, x) \geq 0$ - sought solutions.

We will study the properties of solutions of problem (1), (2) on the basis of a self-similar analysis of solutions of the system of equations constructed by the method of nonlinear splitting and standard equations.

We note that the substitution in (1)

$$
\begin{gathered}
u_{1}(t, x)=e^{-k_{1} t} v_{1}(\tau(t), x), \\
u_{2}(t, x)=e^{-k_{2} t} v_{2}(\tau(t), x)
\end{gathered}
$$

will bring it to mind:

$$
\left\{\begin{array}{l}
\frac{\partial v_{1}}{\partial \tau}=\nabla\left(D_{1} v_{2}^{m_{1}-1}\left|\nabla v_{1}^{k}\right|^{p-2} \nabla v_{1}\right)+k_{1} e^{\left[\beta_{1} k_{1}-(p-2) k k_{1}-\left(m_{1}-1\right) k_{2}\right] t} v_{1}^{\beta_{1}+1}, \\
\frac{\partial v_{2}}{\partial \tau}=\nabla\left(D_{2} v_{1}^{m_{2}-1}\left|\nabla v_{2}^{k}\right|^{p-2} \nabla v_{2}\right)+k_{2} e^{\left[\beta_{2} k_{2}-(p-2) k k_{2}-\left(m_{2}-1\right) k_{1}\right] t} v_{2}^{\beta_{2}+1},
\end{array}\right.
$$

$$
\left.v_{1}\right|_{t=0}=v_{10}(x),\left.v_{2}\right|_{t=0}=v_{20}(x) .
$$

If $k_{1}\left[(p-2) k-\left(m_{1}+1\right)\right]=k_{2}\left[(p-2) k-\left(m_{2}+1\right)\right]$, then choosing

$$
\tau(t)=\frac{e^{\left[\left(m_{1}-1\right) k_{2}+(p-2) k k_{1}\right] t}}{\left(m_{1}-1\right) k_{2}+(p-2) k k_{1}}=\frac{e^{\left[\left(m_{2}-1\right) k_{1}+(p-2) k k_{2}\right] t}}{\left(m_{2}-1\right) k_{1}+(p-2) k k_{2}},
$$

we obtain the following system of equations:

$$
\left\{\begin{array}{l}
\frac{\partial v_{1}}{\partial \tau}=\nabla\left(D_{1} v_{2}^{m_{1}-1}\left|\nabla v_{1}^{k}\right|^{p-2} \nabla v_{1}\right)+a_{1}(t) v_{1}^{\beta_{1}+1}, \\
\frac{\partial v_{2}}{\partial \tau}=\nabla\left(D_{2} v_{1}^{m_{2}-1}\left|\nabla v_{2}^{k}\right|^{p-2} \nabla v_{2}\right)+a_{2}(t) v_{2}^{\beta_{2}+1},
\end{array}\right.
$$

Where

$$
\begin{aligned}
& a_{1}=k_{1}\left((p-2) k k_{1}+\left(m_{1}-1\right) k_{2}\right)^{b_{1}}, \\
& b_{1}=\frac{\left(\beta_{1}-(p-2) k\right) k_{1}-\left(m_{1}-1\right) k_{2}}{(p-2) k k_{1}+\left(m_{1}-1\right) k_{2}}, \\
& a_{2}=k_{2}\left(\left(m_{2}-1\right) k_{1}+(p-2) k k_{2}\right)^{b_{2}}, \\
& b_{2}=\frac{\left(\beta_{2}-(p-2) k\right) k_{2}-\left(m_{2}-1\right) k_{1}}{\left(m_{2}-1\right) k_{1}+(p-2) k k_{2}} .
\end{aligned}
$$

If $b_{i}=0$, and $a_{i}(t)=$ const $, i=1,2$, then the system has the form:

$$
\left\{\begin{array}{l}
\frac{\partial v_{1}}{\partial \tau}=\nabla\left(D_{1} v_{2}^{m_{1}-1}\left|\nabla v_{1}^{k}\right|^{p-2} \nabla v_{1}\right)+a_{1} v_{1}^{\beta_{1}+1} \\
\frac{\partial v_{2}}{\partial \tau}=\nabla\left(D_{2} v_{1}^{m_{2}-1}\left|\nabla v_{2}^{k}\right|^{p-2} \nabla v_{2}\right)+a_{2} v_{2}^{\beta_{2}+1}
\end{array}\right.
$$

Below, we describe one of the methods for obtaining a self-similar system for the system of equations (5). It consists in the following. We first find a solution of the system of ordinary differential equation

$$
\left\{\begin{array}{l}
\frac{d \bar{v}_{1}}{d \tau}=-a_{1} \bar{v}_{1}^{\beta_{1}+1} \\
\frac{d \bar{v}_{2}}{d \tau}=-a_{2} \bar{v}_{2}^{\beta_{2}+1}
\end{array}\right.
$$

in the form

$$
\bar{v}_{1}(\tau)=(\tau(t))^{-\gamma_{1}}, \gamma_{1}=\frac{1}{\beta_{1}}, \quad \bar{v}_{2}(\tau)=(\tau(t))^{-\gamma_{2}}, \gamma_{2}=\frac{1}{\beta_{2}},
$$

for the case $b_{i}=0$, and $a_{i}(t)=$ const $, i=1,2$. And in the case of $b_{i} \neq 0$, and $a_{i}(t)=$ const $, i=1,2$ we find a solution of the system of ordinary differential equations 


$$
\left\{\begin{array}{l}
\frac{d \bar{v}_{1}}{d \tau}=-a_{1} \tau^{b_{1}} \bar{v}_{1}^{\beta_{1}+1} \\
\frac{d \bar{v}_{2}}{d \tau}=-a_{2} \tau^{b_{2}} \bar{v}_{2}^{\beta_{2}+1}
\end{array}\right.
$$

Then the solution of system (5) is sought in the form

$$
\begin{aligned}
& v_{1}(t, x)=\bar{v}_{1}(\tau) w_{1}(\tau(t), x), \\
& v_{2}(t, x)=\bar{v}_{2}(\tau) w_{2}(\tau(t), x),
\end{aligned}
$$

intheform

$$
\begin{gathered}
\bar{v}_{2}(\tau)=(\tau(t))^{-\gamma_{1}}, \gamma_{1}=\frac{b_{1}+1}{\beta_{1}} \\
\bar{v}_{2}(\tau)=(\tau(t))^{-\gamma_{2}}, \gamma_{2}=\frac{b_{2}+1}{\beta_{2}}
\end{gathered}
$$

And $\tau=\tau(t)$ is chosen so

Then for $z_{i}(\tau, \varphi(|x|)), i=1,2$ we obtain a system of equations:

$$
\left\{\begin{array}{l}
\frac{\partial w_{1}}{\partial \tau}=\nabla\left(D_{1} w_{2}^{m_{1}-1}\left|\nabla w_{1}^{k}\right|^{p-2} \nabla w_{1}\right)+\psi_{1}\left(w_{1}-w_{1}^{\beta_{1}+1}\right) \\
\frac{\partial w_{2}}{\partial \tau}=\nabla\left(D_{2} w_{1}^{m_{2}-1}\left|\nabla w_{2}^{k}\right|^{p-2} \nabla w_{2}\right)+\psi_{2}\left(w_{2}-w_{2}^{\beta_{2}+1}\right)
\end{array}\right.
$$

where

$$
\begin{gathered}
\psi_{1}= \begin{cases}\frac{1}{\left(1-\left[\gamma_{1}(p-2) k+\gamma_{2}\left(m_{1}-1\right)\right]\right) \tau}, & \text { if } 1-\left[\gamma_{1}(p-2) k+\gamma_{2}\left(m_{1}-1\right)>0,\right. \\
\left.\gamma_{1} c_{1}^{-\left(1-\gamma_{1}(p-2) k+\gamma_{2}\left(m_{1}-1\right)\right]}\right), & \text { if } 1-\left[\gamma_{1}(p-2) k+\gamma_{2}\left(m_{1}-1\right)=0,\right.\end{cases} \\
\psi_{2}= \begin{cases}\frac{1}{\left(1-\left[\gamma_{2}(p-2)+\gamma_{1}\left(m_{2}-1\right)\right]\right) \tau}, & \text { if } 1-\left[\gamma_{2}(p-2)+\gamma_{1}\left(m_{2}-1\right)\right]>0, \\
\gamma_{2} c_{1}^{-\left(1-\left[\gamma_{2}(p-2)+\gamma_{1}\left(m_{2}-1\right)\right]\right)}, & \text { if } 1-\left[\gamma_{2}(p-2)+\gamma_{1}\left(m_{2}-1\right)\right]=0 .\end{cases}
\end{gathered}
$$

If $1-\left[\gamma_{1}(p-2) k+\gamma_{2}\left(m_{1}-1\right)=0\right.$, self-similar solution of system (9) has the form

$$
w_{i}(\tau(t), x)=f_{i}(\xi), i=1,2, \xi=x /[\tau(t)]^{1 / p}, \quad .
$$

Then substituting (10) in (8) with respect to $f_{i}(\xi)$ we obtain a system of self-similar equations 
Estimation of the Solution of the Kolmogorov-Fisher Type Biological Population Task by Taking Into Account the Reaction-Diffusion

$$
\left\{\begin{array}{l}
\xi^{1-N} \frac{d}{d \xi}\left(\xi^{N-1} f_{2}^{m_{1}-1}\left|\frac{d f_{1}^{k}}{d \xi}\right|^{p-2} \frac{d f_{1}}{d \xi}\right)+\frac{\xi}{p} \frac{d f_{1}}{d \xi}+\mu_{1} f_{1}\left(1-f_{1}^{\beta_{1}}\right)=0, \\
\xi^{1-N} \frac{d}{d \xi}\left(\xi^{N-1} f_{1}^{m_{2}-1}\left|\frac{d f_{2}^{k}}{d \xi}\right|^{p-2} \frac{d f_{2}}{d \xi}\right)+\frac{\xi}{p} \frac{d f_{2}}{d \xi}+\mu_{2} f_{2}\left(1-f_{2}^{\beta_{2}}\right)=0 .
\end{array}\right.
$$

where $\mu_{1}=\frac{1}{\left(1-\left[\gamma_{1} k(p-2)+\gamma_{2}\left(m_{1}-1\right)\right]\right)}$ and $\mu_{2}=\frac{1}{\left(1-\left[\gamma_{2} k(p-2)+\gamma_{1}\left(m_{2}-1\right)\right]\right)}$.

The system (11) has an approximate solution of the form

$$
\bar{f}_{1}=A\left(a-\xi^{\gamma}\right)^{n_{1}}, \gamma=p /(p-1), \bar{f}_{2}=B\left(a-\xi^{\gamma}\right)^{n_{2}},
$$

Where A and B constant and

$$
n_{1}=\frac{(k(p-2)+1)\left(k(p-2)-\left(m_{1}+1\right)\right)}{k^{2}(p-2)^{2}-\left(m_{1}-1\right)\left(m_{2}-1\right)}, n_{2}=\frac{(k(p-2)+1)\left(k(p-2)-\left(m_{2}+1\right)\right)}{k^{2}(p-2)^{2}-\left(m_{1}-1\right)\left(m_{2}-1\right)}
$$

In this paper, based on the method described above, the qualitative properties of the solutions of the system (1) are investigated, and on this basis the problem of choosing the initial approximation for the iterative solution is solved, leading to rapid convergence to the solution of the Cauchy problem (1), (2), depending on the value of the numerical parameters and initial data. For this purpose, the asymptotic representation of the solution found by us was used as the initial approximation. This allowed us to perform a numerical experiment and visualization of the process, described by the system (1), depending on the values entering into the system of numerical parameters.

\section{BUILDING THE UPPER SOLUTION}

We now consider the construction of the upper solution for the system (11).

We note that the functions $\bar{f}_{1}(\xi), \bar{f}_{2}(\xi)$ have properties

$$
\begin{aligned}
& \bar{f}_{2}^{m_{1}-1}\left|\frac{d \bar{f}_{1}^{k}}{d \xi}\right|^{p-2} \frac{d \bar{f}_{1}}{d \xi}=-A^{k(p-2)+1} B^{m_{2}-1}\left(\gamma \gamma_{1}\right)^{k(p-2)+1} \xi \bar{f}_{1} \in C(0, \infty) \\
& \bar{f}_{1}^{m_{2}-1}\left|\frac{d \bar{f}_{2}^{k}}{d \xi}\right|^{p-2} \frac{d \bar{f}_{2}}{d \xi}=-A^{m_{2}-1} B^{k(p-2)+1}\left(\gamma \gamma_{2}\right)^{k(p-2)+1} \xi \bar{f}_{2} \in C(0, \infty)
\end{aligned}
$$

and

$$
\left\{\begin{array}{l}
\xi^{1-N} \frac{d}{d \xi}\left(\xi^{N-1} \bar{f}_{2}^{m_{1}-1}\left|\frac{d \bar{f}_{1}^{k}}{d \xi}\right|^{p-2} \frac{d \overline{f_{1}}}{d \xi}\right)=-\left|\gamma \gamma_{1}\right|^{k(p-2)+1} \gamma \gamma_{1} A^{k(p-2)+1} B^{m_{1}-1}\left(N \overline{f_{1}}+\xi \frac{d \overline{f_{1}}}{d \xi}\right) \\
\xi^{1-N} \frac{d}{d \xi}\left(\xi^{N-1} \bar{f}_{1}^{m_{2}-1}\left|\frac{d f_{2}^{k}}{d \xi}\right|^{p-2} \frac{d f_{2}}{d \xi}\right)=-\left|\gamma \gamma_{2}\right|^{k(p-2)+1} \gamma \gamma_{2} A^{m_{2}-1} B^{k(p-2)+1}\left(N \bar{f}_{2}+\xi \frac{d \bar{f}_{2}}{d \xi}\right)
\end{array}\right.
$$

We choose A and B from the system of nonlinear algebraic equations 


$$
\begin{aligned}
& \left|\gamma \gamma_{1}\right|^{k(p-2)+1} \gamma \gamma_{1} A^{k(p-2)+1} B^{m_{1}-1}=1 / p \\
& \left|\gamma \gamma_{2}\right|^{k(p-2)+1} \gamma \gamma_{2} A^{m_{2}-1} B^{k(p-2)+1}=1 / p
\end{aligned}
$$

Then the functions $\bar{f}_{1}, \bar{f}_{2}$ are solutions of the Zel'dovich-Kompaneets type for the system (1) and in the domain $|\xi|<(a)^{(p-1) / p}$ they satisfy the system of equations

$$
\left\{\begin{array}{l}
\xi^{1-N} \frac{d}{d \xi}\left(\xi^{N-1} \bar{f}_{2}^{m_{1}-1}\left|\frac{d \bar{f}_{1}^{k}}{d \xi}\right|^{p-2} \frac{d \bar{f}_{1}}{d \xi}\right)+\frac{\xi}{p} \frac{d \bar{f}_{1}}{d \xi}+\frac{N}{p} \bar{f}_{1}=0 \\
\xi^{1-N} \frac{d}{d \xi}\left(\xi^{N-1} \bar{f}_{1}^{m_{2}-1}\left|\frac{d \bar{f}_{2}^{k}}{d \xi}\right|^{p-2} \frac{d \bar{f}_{2}}{d \xi}\right)+\frac{\xi}{p} \frac{d \bar{f}_{2}}{d \xi}+\frac{N}{p} \bar{f}_{2}=0
\end{array}\right.
$$

in the classical sense.

Due to the fact that

$$
\left\{\begin{array}{l}
\xi^{N-1} \bar{f}_{2}^{m_{1}-1}\left|\frac{d \bar{f}_{1}^{k}}{d \xi}\right|^{p-2} \frac{d \bar{f}_{1}}{d \xi}=\xi^{N} \bar{f}_{1} \\
\xi^{N-1} \bar{f}_{1}^{m_{2}-1}\left|\frac{d \bar{f}_{2}^{k}}{d \xi}\right|^{p-2} \frac{d \bar{f}_{2}}{d \xi}=\xi^{N} \bar{f}_{2}
\end{array}\right.
$$

function $\bar{f}_{1}(\xi), \bar{f}_{2}(\xi)$ and the flows have the following smoothness property

$$
\begin{gathered}
0 \leq \bar{f}_{1}(\xi), \xi^{N-1} \bar{f}_{2}^{m_{1}-1}\left|\frac{d \bar{f}_{1}^{k}}{d \xi}\right|^{p-2} \frac{d \bar{f}_{1}}{d \xi}=\xi^{N} \bar{f}_{1} \in C(0, \infty), \\
0 \leq \bar{f}_{2}(\xi), \xi^{N-1} \bar{f}_{1}^{m_{2}-1}\left|\frac{d \bar{f}_{2}^{k}}{d \xi}\right|^{p-2} \frac{d \bar{f}_{2}}{d \xi}=\xi^{N} \bar{f}_{2} \in C(0, \infty) .
\end{gathered}
$$

We choose A and B so that the inequalities carried out

$$
\begin{aligned}
& \left|\gamma \gamma_{1}\right|^{k(p-2)+1} \gamma \gamma_{1} A^{k(p-2)+1} B^{m_{1}-1} \geq 1 / p \\
& \left|\gamma \gamma_{2}\right|^{k(p-2)+1} \gamma \gamma_{2} A^{m_{2}-1} B^{k(p-2)+1} \geq 1 / p
\end{aligned}
$$

Then, since 


$$
\left\{\begin{array}{l}
\xi^{1-N} \frac{d}{d \xi}\left(\xi^{N-1} \bar{f}_{2}^{m_{1}-1}\left|\frac{d \bar{f}_{1}^{k}}{d \xi}\right|^{p-2} \frac{d \bar{f}_{1}}{d \xi}\right)+\frac{\xi}{p} \frac{d \bar{f}_{1}}{d \xi}=-\frac{N}{p} \bar{f}_{1} \\
\xi^{1-N} \frac{d}{d \xi}\left(\xi^{N-1} \bar{f}_{1}^{m_{2}-1}\left|\frac{d \bar{f}_{2}^{k}}{d \xi}\right|^{p-2} \frac{d \bar{f}_{2}}{d \xi}\right)+\frac{\xi}{p} \frac{d \bar{f}_{2}}{d \xi}=-\frac{N}{p} \bar{f}_{2}
\end{array}\right.
$$

That by virtue of the fact that

$$
\frac{d \bar{f}_{1}}{d \xi} \leq 0, \frac{d \bar{f}_{2}}{d \xi} \leq 0 \text { npu } \xi \in(0, \infty)
$$

from (12) we have

$$
\begin{aligned}
& \xi^{1-N} \frac{d}{d \xi}\left(\xi^{N-1-} \bar{f}_{2}^{m_{1}-1}\left|\frac{d \bar{f}_{1}^{k}}{d \xi}\right|^{p-2} \frac{d \bar{f}_{1}}{d \xi}\right)+\frac{\xi}{p} \frac{d \bar{f}_{1}}{d \xi} \leq 0, \\
& \xi^{1-N} \frac{d}{d \xi}\left(\xi^{N-1} \bar{f}_{1}^{m_{1}-1}\left|\frac{d \bar{f}_{2}^{k}}{d \xi}\right|^{p-2} \frac{d \bar{f}_{2}}{d \xi}\right)+\frac{\xi}{p} \frac{d \bar{f}_{2}}{d \xi} \leq \mathrm{O}, \\
& \xi \in(0, \infty)
\end{aligned}
$$

Then in the region $Q \quad$ according to the principle of comparing solutions we have

Theorem 1. Let $u_{i}(0, x) \leq u_{i \pm}(0, x), x \in R$

Then for the solution of the problem (1) in the domain $\Omega$ we have the estimate

$$
\begin{aligned}
& u_{1}(t, x) \leq u_{1+}(t, x)=e^{k_{1} t} \tau^{-\alpha_{1}} \bar{f}_{1}(\xi), \\
& u_{2}(t, x) \leq u_{2+}(t, x)=e^{k_{2} t} \tau^{-\alpha_{2}} \bar{f}_{2}(\xi), \\
& \xi=x /[\tau(t)]^{1 / p}
\end{aligned}
$$

where $\bar{f}_{1}(\xi), \bar{f}_{2}(\xi) u \tau(t)$ - the functions defined above.

\section{CONCLUSION}

Thus, the proposed nonlinear mathematical model of a biological population with a double nonlinearity correctly reflects the process under study. Carrying out the analysis of the results on the basis of the obtained estimates of the solutions gives an exhaustive picture of the process in two-component competing systems of the biological population with preservation of the localization properties in the final range and the size of the outbreak. It makes it possible to estimate the propagation velocity of diffusion waves.

\section{REFERENCES}

1. Aripov M. Method of Reference Equations for Solving Nonlinear Boundary-Value Problems Tashkent, Fan, 1988, 137 p.

2. Belotelov N.V, Lobanov A.I Population models with nonlinear diffusion. // Math modeling. -M .; 1997, No. 12, pp. 43-56.

3. Gauze G.F. On the processes of destruction of one species by another in populations of infusorians // Zoological Journal, 1934, Vol. 13, No. 1.

4. Aripov M., Muhammadiev J. Asymptotic behaviour of automodel solutions for one system of quasilinear equations of parabolic type. BuletinStiintific-UniversitateadinPitesti, $\quad$ Seria Matematica si Informatica. N 3. 1999. pg. 19-40

5. Aripov M.M. Muhamediyeva D.K. To the numerical modeling of self-similar solutions of reaction-diffusion system of the one task of biological population of Kolmogorov-Fisher type. International Journal of Engineering and Technology. Vol-02, Iss-11, Nov-2013. 2013.

6. Aripov M.M. Mukhamedieva D.K Approaches to solving one task of a biological population. Questions of computational and applied mathematics. -Tashkent. 2013. Issue 129. -C.22-31.Aripov M.M. Mukhamedieva DK Approaches to solving one task of a biological population. Questions of computational and applied mathematics. Tashkent. 2013. Issue 129.

7. Murray J. Nonlinear diffusion equations in biology. M., Mir, 1983, 397 pages.

8. E. E. Holmes, M. A. Lewis, J. E. Banks, and R. R. Veit. Partial differential equations in ecology: Spatial interactions and population dynamics. Ecology, 75:17-29, 1994. 
9. J. Huisman, J. Sharples, J. M. Stroom, P. M. Visser, W. E. A. Kardinaal, J. M. H. Verspagen, and B. Sommeijer. Changes in turbulent mixing shift competition for light between phytoplankton species. Ecology, $85: 2960-2970, \quad 2004$ 